(e-migrinter

e-Migrinter

12 | 2014

Les chiffres de l'immigration : un regard critique sur les statistiques

\title{
Une étrange absence. La faible prise en compte des sorties dans les statistiques migratoires
}

Luc Legoux et Renaud Orain

\section{(2) OpenEdition}

\section{Journals}

Édition électronique

URL : https://journals.openedition.org/e-migrinter/389

DOI : 10.4000/e-migrinter.389

ISSN : 1961-9685

Éditeur

UMR 7301 - Migrinter

Édition imprimée

Date de publication : 15 octobre 2014

Pagination : 52-61

ISSN : 1961-9685

\section{Référence électronique}

Luc Legoux et Renaud Orain, « Une étrange absence. La faible prise en compte des sorties dans les statistiques migratoires », e-Migrinter [En ligne], 12 | 2014, mis en ligne le, consulté le 20 mai 2021. URL : http://journals.openedition.org/e-migrinter/389; DOI : https://doi.org/10.4000/e-migrinter.389 


\title{
Une étrange absence. La faible prise en compte des sorties dans les statistiques migratoires
}

\author{
Luc Legoux et Renaud Orain
}

Figure $\mathbf{n}^{\circ} \mathbf{1}$ : Une divergence croissante.
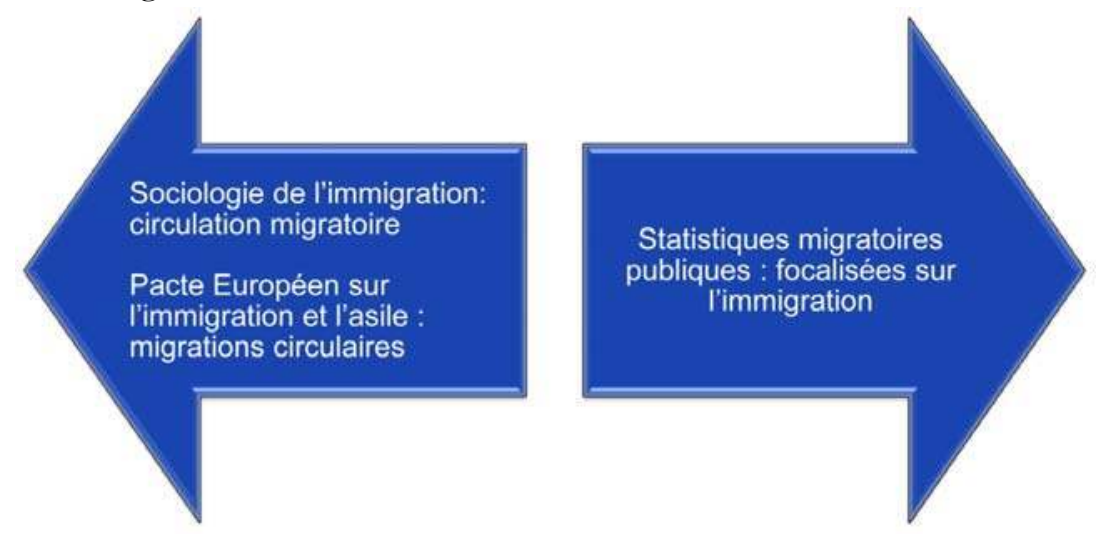

Source : Luc Legoux.

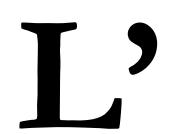

objectif de cette
communication est
d'explorer la quasi

absence de l'émigration dans les statistiques publiques françaises.

Les sorties du territoire, sans

lesquelles il ne peut exister de migrations circulaires, sont laissées dans l'ombre alors même que le concept de « migrations circulaires ", étudié au sein du laboratoire Migrinter à la fin des années 1990 (Ma Mung, Doraï et alii, 1998), est réintroduit dans le milieu scientifique et politique ${ }^{1}$.
La circulation migratoire est en effet au cœur de très nombreux travaux de géographes et sociologues et la migration circulaire est promue par la politique migratoire européenne telle qu'elle est énoncée dans le pacte européen sur l'immigration et l'asile élaboré sous la présidence française de l'Union européenne en $2008^{2}$. L'absence de l'émigration ne peut être sans conséquence sur la perception des flux migratoires par l'opinion publique française.

Figure $\mathbf{n}^{\circ} 2$ : Conséquences.

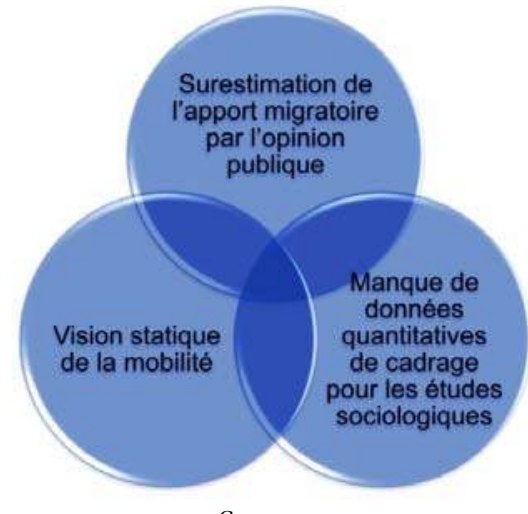

Source : auteurs.

${ }^{2}$ Disponible sur Internet]
${ }^{1}$ Secrétariat général du comité interministériel de contrôle de l'immigration (2012) Les chiffres de la politique de l'immigration et de l'intégration (rapport au parlement), pp.169 et 201. Disponible sur Internet] 
Les principales conséquences de cette absence de statistiques de sortie, qu'elle soit voulue ou non, sont une forte surestimation de l'apport migratoire à la croissance de la population, et une certaine confusion entre les chiffres du solde migratoire et ceux des flux d'immigration. En effet, publier de nombreuses statistiques sur les flux d'entrée sur le territoire sans faire de même pour les flux de sortie conduit à ce que l'opinion publique ignore ces flux de sortie qui ne sont pourtant pas négligeables dans les mouvements de la population. Cette méconnaissance des sorties n'est certainement pas étrangère au sentiment d'invasion qui alimente la xénophobie ${ }^{3}$. Le déficit d'informations sur les flux de retour induit également une vision statique de la mobilité où toute immigration est considérée comme «permanente». Enfin, dans le domaine scientifique, le manque de statistiques publiques sur l'émigration contribue au relatif désintérêt de la recherche pour cette dimension importante de la mobilité.

Tableau $\mathbf{n}^{\circ} \mathbf{1}$ : Les statistiques de sortie sur internet.

\begin{tabular}{|cc|}
\hline $\begin{array}{c}\text { Institutions } \\
\text { présentes sur } \\
\text { internet }\end{array}$ & Données \\
\hline $\begin{array}{c}\text { Ministère en charge } \\
\text { de l'immigration }\end{array}$ & $\begin{array}{c}\text { Mesures d'éloignement à } \\
\text { partir du territoire } \\
\text { métropolitain }\end{array}$ \\
\hline Insee & Soldes migratoires \\
\hline Ined & Aucune \\
\hline
\end{tabular}

L'accès public aux statistiques sur l'émigration est très réduit. L'existence d'un ministère de l'immigration de 2007 à 2012 n'a pas amélioré la diffusion, voire le recueil de l'information sur ce sujet. Ce ministère affichait même un glossaire qui ignorait le mot "émigré ». Les seules statistiques de sortie se résument à celles des mesures d'éloignement du territoire, ce qui renforce la vision d'une immigration qui ne repart que sous la contrainte.

Dans l'optique officielle française, l'immigré est une personne présente en France et née de nationalité étrangère à l'étranger. Les personnes nées françaises à l'étranger ne sont donc pas comptabilisées avec les immigrés lorsqu'elles immigrent en France. Ceci ne simplifie pas les statistiques et surtout, révèle une perception identitaire des flux migratoires. L'Institut national de la statistique et des études économiques (Insee) réalise pourtant une estimation des soldes migratoires annuels, ces chiffres résultent des mouvements d'entrées et de sorties des étrangers et des Français. Même si l'estimation de ces soldes est délicate, il s'agit d'un indicateur fondamental. Quant aux rubriques sur les flux migratoires du site de l'Institut national d'études démographiques (Ined), elles se limitent aux «Flux d'immigration» et aux «Immigrés et étrangers ». Les sorties y sont donc totalement ignorées.

\footnotetext{
${ }^{3}$ Le 17 mars 2011, Claude Guéant déclarait ainsi sur Europe 1 que les Français à force d'immigration incontrôlée ont parfois le sentiment de ne plus être chez. eux, ou bien ils ont le sentiment de voir des pratiques qui s'imposent à eux et qui ne correspondent pas aux règles de notre vie sociale.
} 
Tableaux n² \& 3 : Mesures d'éloignement à partir du territoire métropolitain en 2009 et 2010.

\begin{tabular}{|c|c|c|c|}
\hline Mesures 2009 & Prononcées & Exécutées & Taux en \% \\
\hline $\begin{array}{c}\text { Interdictions du territoire } \\
\text { (ITF) }\end{array}$ & 2009 & 113 & 66,2 \\
\hline $\begin{array}{c}\text { Arrêtés préfectoraux de } \\
\text { reconduite à la frontière } \\
\text { (APRF) }\end{array}$ & 40116 & 10422 & 26,0 \\
\hline Arrêtés d'expulsion & 215 & 198 & 92,1 \\
\hline Décisions de réadmission & 12162 & 4156 & 34,2 \\
\hline $\begin{array}{c}\text { Obligations de quitter le } \\
\text { territoire français (OQTF) }\end{array}$ & 40191 & 4914 & 12,2 \\
\hline Retours aidés & Sans objet & 8268 & Sans objet \\
\hline Totaux & 94693 & 29288 & 30,9 \\
\hline
\end{tabular}

\begin{tabular}{|c|c|c|}
\hline Mesures 2009 & Prononcées & Exécutées \\
\hline $\begin{array}{l}\text { Interdictions du territoire } \\
\text { (ITF) }\end{array}$ & 1683 & 1201 \\
\hline $\begin{array}{l}\text { Arrêtés préfectoraux de } \\
\text { reconduite à la frontière } \\
\text { (APRF) }\end{array}$ & 32519 & 9370 \\
\hline Arrêtés d'expulsion & 212 & 164 \\
\hline Décisions de réadmission & 10849 & 3504 \\
\hline $\begin{array}{l}\text { Obligations de quitter le } \\
\text { territoire français (OQTF) }\end{array}$ & 39083 & 5383 \\
\hline Retours aidés & Sans objet & 8404 \\
\hline Totaux & 84346 & 28026 \\
\hline
\end{tabular}

Les éloignements du territoire métropolitain concernent un peu moins de 30000 personnes par an. C'est un indicateur imparfait pour deux raisons. D'une part, les réadmissions simplifiées, c'est-à-dire les renvois sans délai ni formalité lors de l'interpellation d'un étranger qui a franchi illégalement la frontière, ne sont pas comptabilisées (9 255 en 2010). D’autre part, les retours volontaires aidés sont comptabilisés alors qu'il ne s'agit pas d'éloignements toujours forcés.

\section{Les mesures d'éloignement} prononcées ne sont exécutées que dans moins d'un quart des cas. Le rapport du Comité interministériel de contrôle de l'immigration (CICI) commet à ce propos une erreur flagrante et répétée. Le taux d'exécution des mesures est mathématiquement le nombre de mesures exécutées divisé par le nombre de mesures prononcées, or le Cici intègre les retours aidés dans le nombre de mesures exécutées mais évidemment pas dans les mesures prononcées où cette rubrique est, assez logiquement, notée «sans objet». Il en résulte une forte surestimation du taux d'exécution.

Pour 2009, le taux erroné de 30,9\% est affiché dans le tableau (alors que le taux réel est de 22,2\%). Cette erreur a été signalée publiquement. Pour 2010, le calcul erroné est toujours le même mais l'artifice est moins visible puisque le pourcentage erroné n'est plus dans le tableau mais dans le texte : «Le taux d'exécution global toutes mesures confondues a progressé légèrement en 2010 par rapport à l'année précédente, passant de $30,9 \%$ à $33,2 \%$ soit une hausse de 2,3 points » ( $8^{\text {ème }}$ Rapport CICI, p. 74). En réalité, le taux est de (28026$8404) / 84346=23,26 \%$ soit une hausse de 1,07 point par rapport à 2009, et une surestimation de 10 points par rapport à la réalité. En 2010, on remarque qu'il y a 42 retours volontaires aidés pour 100 
éloignements forcés. Enfin, malgré la liberté de circulation au sein de l'UE, les Roumains représentent 29,9\% de l'ensemble des éloignements (éloignement juridiquement possible pour des motifs liés à divers troubles à l'ordre public selon les autorités).

Figure $\mathbf{n}^{\circ} \mathbf{3}$ : Soldes migratoires Insee (20032011). internet de l'Ined où on peut lire : «À noter qu'il n'existe pas de statistiques fiables sur les émigrations (ou départs). L'Insee publie néanmoins chaque année une estimation du solde migratoire ${ }^{4}$. Cette citation est une critique à peine voilée des travaux de l'Insee. En effet, sans statistiques fiables sur les sorties, comment les soldes entrées-sorties pourraient-ils être fiables?

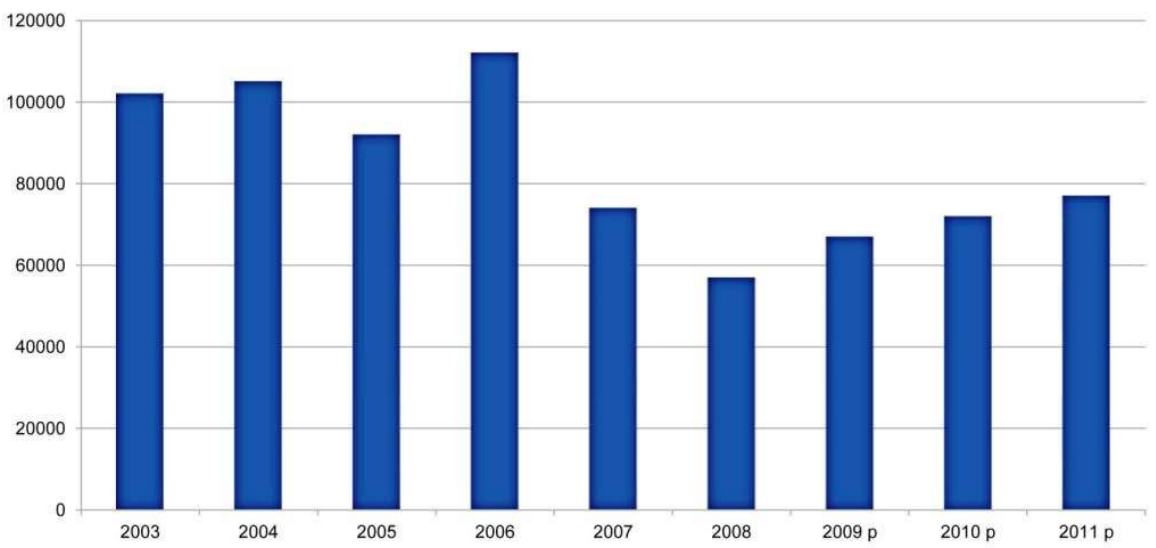

Source : Insee, réalisation des auteurs.

Le solde migratoire, après avoir atteint 112000 en 2006 puis baissé jusqu'à 57000 en 2008, est estimé provisoirement à 77000 en 2011. Ce solde est la différence entre les entrées et les sorties d'étrangers et de Français. On ne peut donc comparer utilement ces soldes aux nombres d'entrées annuelles d'étrangers publiés par ailleurs, sans connaitre, au minimum, les nombres d'entrées et de sorties de Français. La fiabilité de ces soldes est mise en cause sur le site plus loin).

\begin{tabular}{|c|c|c|c|c|}
\hline Année & $\begin{array}{c}\text { Population au } \\
\text { 1erianvier }\end{array}$ & Solde naturel & $\begin{array}{c}\text { Solde migratoire } \\
\text { évalué }\end{array}$ & Ajustement \\
\hline 1989 & 57659542 & +258574 & +78285 & 0 \\
\hline 1990 & 57996401 & +258685 & +77393 & -52344 \\
\hline 1991 & 58280135 & + 257191 & +88397 & -54486 \\
\hline 1992 & 58571237 & +244941 & +89349 & -53525 \\
\hline 1993 & 58852002 & +200773 & +70340 & -53038 \\
\hline 1994 & 59070077 & +212653 & +51301 & -53454 \\
\hline 1995 & 59280577 & +218745 & +42193 & -54102 \\
\hline 1996 & 59487413 & +219425 & +38241 & -53902 \\
\hline 1997 & 59691177 & +218117 & +43279 & -53226 \\
\hline 1998 & 59899347 & +224497 & +50228 & -51407 \\
\hline 1999 & 60122665 & +228530 & +62500 & +94455 \\
\hline 2000 & 60508150 & +266804 & +72000 & +94456 \\
\hline 2001 & 60941410 & +262205 & +87000 & +94455 \\
\hline 2002 & 61385070 & +247504 & +97000 & +94456 \\
\hline 2003 & 61824030 & +230577 & +102000 & +94455 \\
\hline 2004 & 62251062 & +279891 & +105128 & +94456 \\
\hline 2005 & 62730537 & +268741 & +92192 & +94647 \\
\hline 2006 & 63186117 & +302432 & +112141 & 0 \\
\hline 2007 & 63600690 & +287543 & +73626 & 0 \\
\hline 2008 & 63961859 & +285829 & +56812 & 0 \\
\hline $2009(p)$ & 64304500 & +276100 & +67000 & 0 \\
\hline $2010(p)$ & 64647600 & +281581 & +72000 & 0 \\
\hline $2011(p)$ & 65001181 & +272000 & +77000 & 0 \\
\hline
\end{tabular}

Source: Insee, réalisation des auteurs.

l'estimation des soldes est un exercice délicat, les soldes migratoires publiés par l'Insee ne sont très

vraisemblablement pas moins fiables que d'autres statistiques publiées par ailleurs par l'Ined, comme les entrées sur le territoire français d'étrangers communautaires (comme nous le verrons

Tableau $\mathrm{n}^{\circ} 4$ : Soldes migratoires et ajustements dans les recensements. 
Le solde migratoire de l'Insee est basé sur les estimations de l'évolution des flux migratoires à partir de données administratives parcellaires.

Le solde migratoire n'est pas la variable d'ajustement entre les recensements et l'évolution du solde naturel mesuré par l'état civil puisque cet ajustement est réalisé à part en fonction de la fiabilité des différents recensements.

L'absence d'enregistrement des sorties $\mathrm{du}$ territoire, en plein accord avec la Déclaration universelle des droits de l'homme qui stipule que «Toute personne a le droit de quitter tout pays, y compris le sien, et de revenir dans son pays ${ }^{5}$. » justifie-til l'absence de statistiques de sorties? On peut estimer que non, lorsque, par ailleurs, les techniques d'estimations et de sondages sont largement mobilisées, y compris par le recensement qui n'est plus exhaustif. Pourquoi ce manque d'estimation? Nous avons déjà évoqué l'effet de surestimation de l'apport migratoire et l'utilisation politique possible. La crainte de considérations partisanes est renforcée lorsqu'on constate que les flux d'entrées sont mieux traités. Avec la construction européenne et la libre circulation des ressortissants de l'Union européenne, ces derniers ne sont plus soumis à l'obligation de titre de séjour et «sortent» ainsi des statistiques exhaustives d'immigration.

Contrairement aux sorties, l'Ined se risque à une estimation de ces entrées d'étrangers communautaires (55000 par an en 2006, 2007 et 2008, le flux total d'entrées sur le territoire dépassant ainsi les 200 000). La fiabilité de ces estimations est-elle meilleure que celle du solde migratoire?
Tableau n ${ }^{\circ} 5$ : Estimations d'entrées pourtant difficiles et donc fragiles.

\begin{tabular}{|c|c|c|}
\hline \multicolumn{2}{|c|}{ Flux d'immigration : Union européenne } \\
\hline Années & $\begin{array}{c}\text { Union } \\
\text { européenne }\end{array}$ & $\begin{array}{c}\text { dont } \\
\text { polonais }\end{array}$ \\
\hline 2006 & 55000 & 2194 \\
\hline 2007 & 55000 & 28201 \\
\hline 2008 & 55000 & 22951 \\
\hline
\end{tabular}

Source: Ined, réalisation des auteurs.

La fragilité des estimations des entrées non enregistrées est mise en évidence ici en confrontant le chiffre global estimé à 55000 par an, aux effectifs estimés par nationalité. L'évolution estimée pour la nationalité polonaise est si forte que l'on peut avoir des doutes sur la compensation exacte par l'évolution des entrées des autres nationalités.

Ce qui est critiqué ici n'est pas la fragilité des estimations d'entrées d'étrangers communautaires - l'exercice est réellement difficile - mais le parti pris d'estimer les entrées, même de manière très approximative, tout en ignorant les sorties, au prétexte qu'elles ne sont pas enregistrées exhaustivement.

Ce défaut de publication des statistiques de sortie est d'autant plus regrettable et lourd de signification que ces statistiques existent.

\section{Estimations des émigrations dans Eurostat : définitions}

"Immigration" denotes the action by which a person establishes his or her usual residence in the territory of a Member State for a period that is, or is expected to be, of at least 12 months, having previously been usually resident in another Member State or a third country. "Immigrant" is a person undertaking an immigration. » (Eurostat)

" "Emigration" denotes the action by which a person, having previously been usually resident in the territory of a Member State, ceases to have his or her usual residence in that Member State for a period that is, or is expected to be, of at least 12 months. 


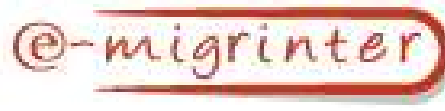

"Emigrant" is a person undertaking an emigration $»^{6}$ (Eurostat).

Les statistiques françaises d'émigrations existent... à l'étranger, sur le site Internet d'Eurostat ${ }^{7}$, organisme européen qui applique les définitions statistiques internationales pour les migrations. Contrairement à la définition française pour laquelle un immigrant est une personne née étrangère à l'étranger, les définitions internationales ne tiennent pas compte de la nationalité mais seulement du changement de résidence pour une durée d'au moins douze mois.
Tous ces chiffres d'immigration et d'émigration sont fournis par l'Insee à Eurostat, ils respectent les définitions qu'Eurostat affiche sur son site et incluent donc les immigrations et émigrations de Français. Le nombre d'émigrants est manifestement calculé par le calcul de la différence entre l'immigration, selon la définition utilisée par Eurostat et le solde estimé arrondi au millier. On remarque que la correction du solde migratoire de 2008 initialement estimé à 76000 et aujourd'hui estimé par l'Insee à 56812 n'est pas prise en compte dans les tableaux Eurostat. Les données 2009 sont absentes (encore en septembre 2012) et les chiffres de 2010 correspondent à une nouvelle série d'estimations ${ }^{8}$.

Tableau $\mathbf{n}^{\circ} \mathbf{6}$ : Les statistiques françaises d'émigration dans Eurostat.

\begin{tabular}{|c|c|c|c|c|c|c|c|c|}
\hline France & 2003 & 2004 & 2005 & 2006 & 2007 & 2008 & 2009 & $2010^{1}$ \\
\hline Immigrations & 236037 & 225629 & 219537 & 219407 & 209781 & 216937 & $X$ & 251159 \\
\hline Émigrations & 134037 & 120629 & 127537 & 107407 & 135781 & 140937 & $X$ & 179159 \\
\hline Solde & 102000 & 10000 & 92000 & 112000 & 74000 & 76000 & & 72000 \\
\hline
\end{tabular}

Source : Eurostat, réalisation des auteurs. 
Tableau $\mathbf{n}^{\circ} 7$ : Les entrées/sorties par nationalité (Français/étrangers)**.

\begin{tabular}{|r|r|r|r|r|}
\hline & 2007 & 2008 & 2009 & $2010 *$ \\
\hline immigration & 209781 & 216937 & $:$ & 251159 \\
\hline dont immigration de français & 63824 & 64049 & 101613 \\
\hline dont immigration d'étranger & 145732 & 152888 & 149546 \\
\hline émigrations & 135781 & 140937 & $:$ & 179159 \\
\hline dont émigration de français & $:$ & 1486 & 116137 \\
\hline dont émigration d'étrangers & $:$ & 139451 & 63022 \\
\hline solde & & & & 72000 \\
\hline solde d'étrangers & & & & 86524 \\
\hline solde de français & & & & -14524 \\
\hline
\end{tabular}

Source : Eurostat, réalisation des auteurs.

*Rupture de série.

**Français sans distinction entre naissance/acquisition/double nationalité.

Tableau $\mathbf{n}^{\circ} \mathbf{8}$ : La cohérence entre les chiffres Eurostat et les statistiques des Français de l'étranger.

\begin{tabular}{|c|c|c|}
\hline $\begin{array}{r}\text { Le MAE dénor } \\
\text { Français enreg } \\
\text { Parmi les e }\end{array}$ & $\begin{array}{l}\text { bre } 1594303 \\
\text { trés fin } 2011 . \\
\text { registrés : }\end{array}$ & $\begin{array}{l}\text { Et évalue à } 500000 \text { les } \\
\text { Français non enregistrés }\end{array}$ \\
\hline $\begin{array}{l}\text { Certains ne sont } \\
\text { pas nés en } \\
\text { France }\end{array}$ & $\begin{array}{c}42,5 \% \text { sont } \\
\text { des binationaux }\end{array}$ & $\begin{array}{c}\text { Courts séjours, } \\
\text { binationaux âgés } \\
\ldots\end{array}$ \\
\hline
\end{tabular}

Source : ministère des Affaires étrangères (MAE) et MFE.

La distinction entre émigration française et étrangère est apparue en 2008 mais avec des résultats peu réalistes (seulement 1486 sorties de Français). Après une interruption des données pour 2009, la nouvelle série à partir de 2010 semble a priori plus réaliste, ce que nous allons vérifier par la suite. Les chiffres de 2010 indiquent un solde migratoire d'étrangers, toutes nationalités confondues, y compris les ressortissants de l'UE (qui ne sont souvent pas perçu sociologiquement comme des immigrés) de +86524 personnes. Le solde migratoire des Français est faiblement négatif :

-14524 personnes.
La population enregistrée est jeune et fortement binationale. Une partie de la population enregistrée n'a pas émigré de France, c'est le cas de certaines personnes nées françaises à l'étranger (binationales ou non). Selon l'enquête 2010 de la Maison des Français de l'étranger (MFE), 11,3\% des enquêtés ne sont pas nés en France, mais cette enquête sous-représente fortement les binationaux. 
Figure $\mathrm{n}^{\circ} 4$ : Évolution de la population française à l'étranger.

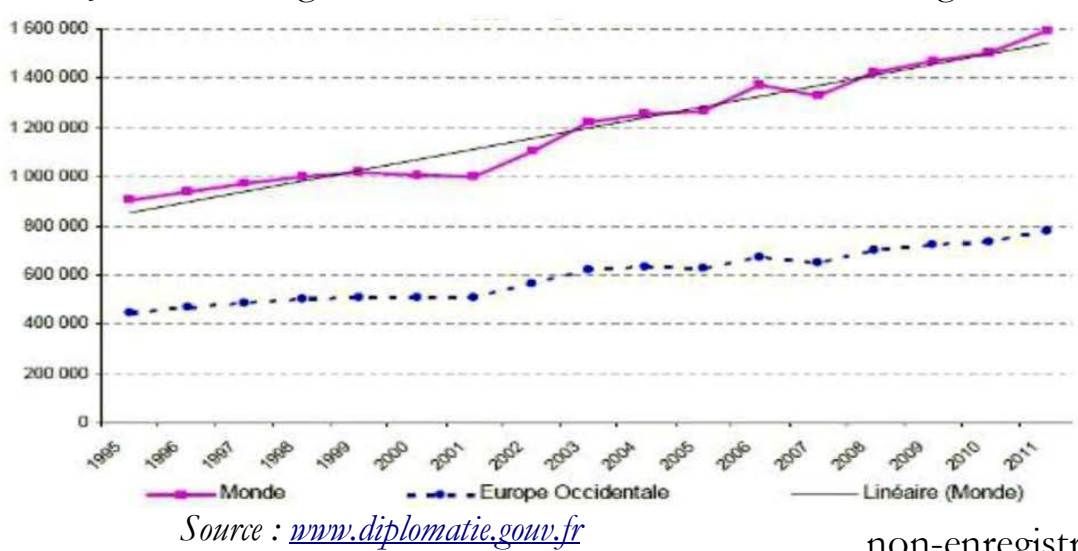

L'évolution de la population inscrite dans le registre des Français à l'étranger résulte de plusieurs facteurs. Les émigrations de Français à l'étranger et leur retour en France, la progression du taux d'enregistrement (sensible les années précédant une élection présidentielle comme 2006 et 2011), les naissances de Français à l'étranger, les abandons d'inscription sans départ vers la France. Les inscriptions devant être renouvelées tous les cinq ans, les départs non déclarés sont connus avec un certain retard'. L'enquête 2010 sur les Français de l'étranger dénombre 115000 nouveaux inscrits en 2010. Avec une progression du nombre d'inscrits de seulement 35000 la même année on en déduit par différence le nombre de 80000 radiations.

Figure $\mathbf{n}^{\circ} \mathbf{5}$ : Immigration: cohérence Insee/registre.
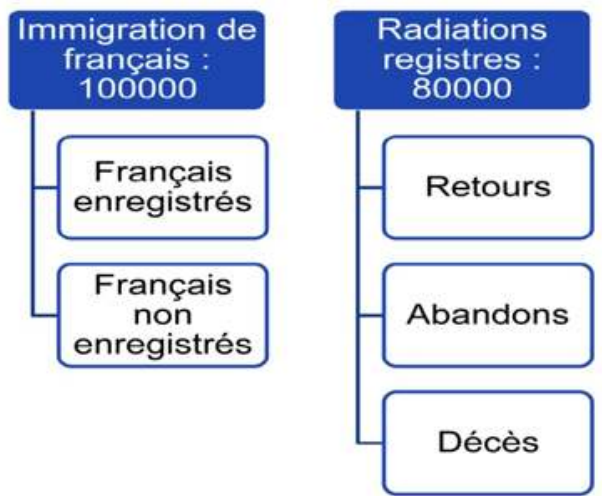

Source : auteur.

9Source : http://www.diplomatie.gouv.fr/
L'immigration en France de Français enregistrés devrait logiquement correspondre aux radiations pour cause de retour (les départs vers d'autres pays correspondent à une radiation dans un pays et à une inscription dans un autre). Pour que les estimations de l'Insee soient cohérentes avec les chiffres $\mathrm{du}$ registre, il faut que l'immigration de Français non-enregistrés soit égale aux nombre d'abandons et de décès plus la différence entre l'immigration de Français et les radiations du registre $(20000)$. Avec une population de 1,5 million relativement jeune, un taux brut de mortalité légèrement inférieur au taux français ( 7 au lieu de 9 pour mille) il y aurait environ 10000 décès. L'immigration de Français non enregistrés serait donc supérieure à 100000 - $80000+$ $10000=30000$ (chiffre que l'on obtient sans aucune radiation par abandon), c'est un peu supérieur à ce qu'on obtient en rapportant le pourcentage de non-inscrits à la population totale des Français établis à l'étranger $(25 \%)$.

Figure $\mathbf{n}^{\circ} \mathbf{6}$ : Émigration : cohérence Insee/registre.

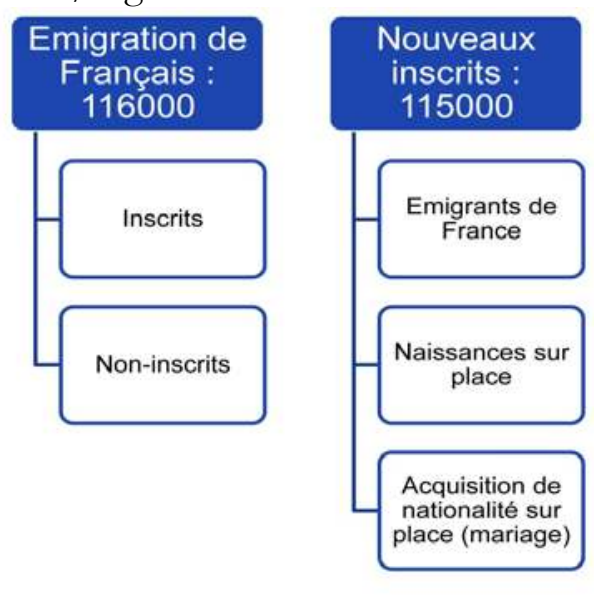

Source: auteur.

Dans la même logique que précédemment, et puisque le nombre d'émigrants estimé par l'Insee est sensiblement égal au nombre de nouveaux 
inscrits, pour que tous les chiffres soient cohérents, il faudrait que le nombre d'émigrants non-inscrits soit égal à la somme des inscriptions pour naissances et des acquisitions de nationalité sur place.

Avec une population de 1,5 million de personnes relativement jeunes et le taux brut de natalité de la France (13 pour mille) le nombre de naissances doit être aux environs de 20000 . On ne connait pas le nombre d'acquisitions de nationalité sur place mais il n'est probablement pas très élevé, ce qui implique que la somme des naissances sur place et des acquisitions de nationalités sur place doit être inférieure à 30000 .

Si le nombre d'émigrations de non-inscrits respectait la proportion de non-inscrits dans la population totale, ce nombre devrait également être inférieur à 30000 , ce qui indique une certaine compatibilité entre les chiffres de l'Insee et les statistiques du registre des Français à l'étranger.

Tableau $\mathbf{n}^{\circ} 9$ : Les immigrants dans les enquêtes « Emploi » (2003-2009). aujourd'hui Adoma). Il se peut que, malgré la connaissance du terrain des enquêteurs grâce à l'échantillon aréolaire, certains enquêtés en habitat précaire ou en situation irrégulière échappent à l'enquête. Il se peut aussi qu'en face d'un enquêteur appartenant à une administration publique (Insee), les enquêtés ne soient poussés à sous-déclarer leurs mobilités géographiques, dans un contexte politique et administratif qui les incite plutôt à faire valoir une présence durable et continue sur le territoire que d'éventuels allers et retours.

L'année 2005 pose problème pour estimer correctement l'immigration de Français (problème non élucidé).

L'année 2005 mise à part, les entrées de Français sont estimées entre 100000 et 130000 par an pour le seul territoire métropolitain. Si l'on veut être cohérent entre ce chiffre et l'idée d'un accroissement du nombre de Français à l'étranger dû à un solde migratoire négatif des Français de la

\begin{tabular}{|c|c|c|}
\hline & \multicolumn{2}{|c|}{ Résidents à l'étranger 12 mois avant } \\
\hline & étrangers & Français \\
\hline 2003 & 132915 & 123271 \\
\hline 2004 & 139671 & 130346 \\
\hline 2005 & 130306 & 88120 \\
\hline 2006 & 147154 & 101311 \\
\hline 2008 & 124773 & 115291 \\
\hline 2009 & 143465 & 106041 \\
\hline Total & 111991 & 133266 \\
\hline & 930275 & 797646 \\
\hline moyenne & 132896 & 113949 \\
\hline
\end{tabular}

France vers le reste du monde, ceci conduit à supposer que l'estimation de 130000 Français partant à l'étranger par an est plutôt une estimation basse. Il n'est pas du tout absurde de penser qu'il puisse y en avoir plus.

Les statistiques d'émigration fournies par l'Insee à Eurostat, mais que nous n'avons pas encore trouvées au 12 septembre 2012 sur le site de l'Insee (sauf concernant les Départements et Régions d'outre-mer et Collectivités d'outre-mer (DOM-COM), comme La Réunion), semblent assez crédibles, et en tout cas cohérentes avec d'autres sources disponibles. On constate que les sorties d'étrangers représentent plus de $40 \%$ des entrées d'étrangers et que la prise en compte du solde migratoire négatif 
des Français explique une croissance du nombre d'immigrés légèrement supérieure aux soldes migratoires totaux. Pourquoi occulter ces informations? L'absence des chiffres de sorties d'étrangers et de migrations de Français dans les nombreux débats politiques sur la maitrise des flux migratoires n'est certainement pas innocente. Cette absence contribue à faire de la migration une question concernant exclusivement l'entrée des étrangers, elle dissimule la circulation migratoire et participe ainsi à la progression de la xénophobie en France.

Luc Legoux Maître de Conférences en Démographie IDUP - Université Paris 1Panthéon Sorbonne, EA CRIDUP $\underline{\text { luc.legoux@,univ-paris1.fr }}$

Renaud Orain Maître de Conférences en Sociologie IDUP - Université Paris 1Panthéon Sorbonne, EA CRIDUP renaud.orain@univ-paris1.fr
Secrétariat général du comité interministériel de contrôle de l'immigration (2012) Les chiffres de la politique de l'immigration et de l'intégration (rapport au parlement), 240 p. Disponible sur Internet]

Simon, Gildas (2008) Migrants et migrations du monde, Paris, La Documentation française, $64 \mathrm{p}$.

\section{Bibliographie}

Le Bras, Hervé (2007) Les 4 mystères de la population française, Paris, Éd. Odile Jacob, 306 p.

Fleurance, Simone (1948) L'émigration française à l'étranger : tendances actuelles, Population, Vol. 3, n³, pp. 561-563.

Ma Mung, Emmanuel; Doraï, Kamel; Loyer, Franz; Hily, Marie-Antoinette (1998) La circulation migratoire, bilan des travaux. Synthèse, Migrations études : revue de synthèse sur l'immigration et la présence étrangère en France, n84, pp. 1-12. 